\title{
Semileptonic B-Meson decays at Belle II
}

\author{
Jo-Frederik Krohn* on behalf of the Belle II collaboration \\ The University of Melbourne, 3010 Parkville, Melborune, Victoria, Australia \\ E-mail: jkrohnestudent.unimelb.edu.au
}

The Belle II experiment is the upgrade of the Belle experiment, performed at the SuperKEKB asymmetric electron-positron-collider, located in Tsukuba, Japan. With an design instantaneous luminosity of $8 \cdot 10^{35} \mathrm{~cm}^{-2} \mathrm{~s}^{-1}$ a dataset of $50 \mathrm{ab}^{-1}$ will be collected. The clean collision environment of the experiment and the large dataset yields the ability to perform high precision measurements of physics mediated by the weak force, such as semileptonic $B$-meson decays. Of special focus of this document are magnitudes of the CKM matrix elements $\mathrm{V}_{\mathrm{ub}}$ and $\mathrm{V}_{\mathrm{cb}}$ measured in $B \rightarrow D^{(\star)} l v$ and $B \rightarrow \pi l v$, where $l=e, \mu$, as well as the ratio of the branching fractions, $R_{D^{(\star)}}:=\mathscr{B}\left(B \rightarrow D^{(\star)} \mu v\right) / \mathscr{B}\left(B \rightarrow D^{(\star)} e v\right)$ and $R_{\tau}:=\mathscr{B}\left(B \rightarrow D^{(\star)} \tau v\right) / \mathscr{B}\left(B \rightarrow D^{(\star)} l v\right)$, of lepton couplings in these decays. Some of these have shown persistent tension with the Standard Model and are therefore of primary interest towards a better understanding of the weak force carriers and potential new physics couplings.

XIV International Conference on Heavy Quarks and Leptons (HQL2018)

May 27- June 1, 2018

Yamagata Terrsa, Yamagata,Japan

${ }^{*}$ Speaker. 


\section{Introduction}

The preceding B-factory experiments Belle and BaBar have been very successful, collecting approximately $1 \mathrm{ab}^{-1}$ and approximately $0.5 \mathrm{ab}^{-1}$ of data respectively, confirming the existence of the theory of $C P$-violation and establishing the Cabibbo-Kobayashi-Maskawa (CKM) matrix as the basis for a theory of flavour, resulting in a Nobel Prize for Kobayashi and Maskawa in 2008. However, many open questions and problems with the Standard Model (SM) remain, such as for example the nature of neutrino masses and the existence and interactions of Dark Matter.

The Belle II experiment takes place at the collision point of $4 \mathrm{GeV}$ positrons with $7 \mathrm{GeV}$ electrons, such that the center of mass energy is slightly above $10.58 \mathrm{GeV}$, the production threshold of the $\Upsilon(4 s)$ resonance. This resonance is used as it decays with $>96 \%$ probability into pairs of $B$ mesons, which then can be used for high precision measurements. The Belle II experiment will collect $50 \mathrm{ab}^{-1}$ of data in total corresponding approximately $5 \cdot 10^{10} \bar{B} B$ pairs.

This will be achieved with an upgrade of the KEKB collider to the SuperKEKB collider which will increase the instantaneous luminosity by a factor of $\approx 40$ by increasing the beam currents by a factor of two and additionally squeezing the volume of the inter action region by a factor of $\approx 20$ [1].

In order to meet the new experimental requirements the detector was upgraded as well. Two layers of pixelated silicon sensors (PXD) together with 4 layers of silicon strip sensors (VXD) will be used for vertex reconstruction. A new central drift chambers (CDC) was constructed to fill the larger outer radius of the tracking volume. A new time of propagation system in the barrel region (TOP) in combination with a particle identification cherenkov detector in the forward region (PID) will yield further improvements for Kaon-Pion separation.

The experimental precision will furthermore be improved by new analysis software methods such as machine learning techniques used in tagging and new approaches to decay tree vertex fitting.

The experiment recorded the first $e^{+} e^{-}$-collision since April $26^{\text {th }} 2018$ with a reduced detector, which will be used for commissioning and Dark Sector analyses. The final experimental setup will be installed in late 2018 allowing operation in March 2019.

\section{Reconstruction of decays with neutrinos in the final state}

Decays with neutrinos in the final state, such as semileptonic decays, are experimentally challenging to reconstruct as the neutrino escapes the detector without interaction. The only signature they leave is missing energy in the calorimeter. Missing energy signatures are hard to reconstruct as they can be easily be mimicked by any particle escaping detection. For example $K_{\mathrm{L}}^{0}$ can easily escape detection because Belle II does not have a hadron calorimeter. Nevertheless, Belle II excels in the reconstruction of this decay type as the experimental environment is much cleaner and the initial state energy is much better known than for example in hadron machines such as the LHC. The experimental observables used in missing energy decays are the residual energy in the calorimeter $E_{\mathrm{ECL}}$ and the missing mass squared

$$
m_{\text {miss }}^{2}=\left(p_{e^{+} e^{-}}-p_{\text {sig }}-p_{\text {tag }}\right)^{2} .
$$


Where the label sig (signal) and tag correspond to the two $B$-mesons present in one event. If a decay is correctly reconstructed there should be little to no energy left in the calorimeter, thus in the $E_{\mathrm{ECL}}$ observable the signal peaks at zero. The same holds for $m_{\text {miss }}^{2}$, as this corresponds to the mass of the reconstructed signal neutrino which is negligibly small and can be considered zero.

There are three approaches to reconstructing $B$-meson events, untagged, hadronically tagged, and a semileptonical tag. The principle idea is to use one of the two $B$-mesons to tag the existence of the other and to constrain the kinematics and flavour.

Untagged approach In the untagged approach one does not reconstruct a second (tag) $B$-meson and instead uses the rest of the event to constrain the 4-vector of the signal- $B$. By summing all 4vectors in the rest of the event one obtains a cone in which the signal $B$-meson must have decayed. If this is not the case for the reconstructed signal candidate one can discard the event. This approach is very efficient but the purity of the reconstructed candidates is low.

Hadronically Tagged In this approach one reconstructs the tag $B$-meson only in hadronic decay modes. The purity of this approach is high compared to the untagged one, however, the efficiency is very low. Due to this fact Belle II uses the Full Event Interpretation (FEI) machine learning technique that reconstructs more than 5000 decay modes [2].

Semileptonically Tagged The semileptonic tagging approach uses purely semileptonic decays modes to reconstruct a second $B$-meson. These have higher branching fractions, but the presence of a neutrino makes the 4-vector reconstruction less precise. More than 200 modes are reconstructed in the FEI in this case.

Further improvements in the tag reconstruction will be made by using a new, global decay chain vertex fitting method, which allows to fit topologies containing neutral particles such as $B \rightarrow$ $D^{\star}\left(D^{0}\left(K^{-} \pi^{+} \pi^{0}\right) \pi\right)$. Established algorithms would fit vertices one by one starting from the bottom of the hierarchy moving towards the $B$-meson so in the given example one would fit $\pi^{0} \rightarrow \gamma \gamma$ and then a vertex from $K^{-} \pi^{+} \pi^{0}$ to reconstruct $D^{0} \rightarrow K^{-} \pi^{+} \pi^{0}$ and so on. The problem that arises with the presence of a $\pi^{0}$ is that since the granularity of the calorimeter is not high enough to provide directional information for the photons one has to assume they originate from the primary interaction point at $\{0,0,0\}$. Otherwise one has not enough constraints to perform the fit of $\pi^{0} \rightarrow \gamma \gamma$. However, this assumption is incorrect - the $D$-meson has a significant flight length in the detector, thus fitting the $\pi^{0}$ to an incorrect vertex position results in an incorrect estimation of the momentum covariance matrix, which then is propagated down the cascade of fits to the $D^{\star}$-meson and $B$-meson. This can be overcome simply by fitting the entire decay chain in one simultaneousness fit. The technical difficulty is that these fits essentially are an inversion of a matrix, which naively scales as $\mathscr{O}\left(n^{3}\right)$ but this can be overcome by using a Kalman Filter.

\section{Measurement of $B \rightarrow D^{(\star)} l v$ and determination of $\left|\mathrm{V}_{\mathrm{cb}}\right|$ and $R_{D^{\star}}$}

Measurements of $B \rightarrow D^{(\star)} l v$ with $l=e, \mu$ allow for the extraction of the CKM matrix element $\left|\mathrm{V}_{\mathrm{cb}}\right|$ as well as the measurement of the ratio of the differential decay rates to different lepton species $R_{D^{\star}}$. The determination of $\left|\mathrm{V}_{\mathrm{cb}}\right|$ has shown tension of $2.5 \sigma$ between inclusive $\left(B \rightarrow X_{c} l v\right.$, 
where $X_{c}$ is any light meson containing a $c$-quark) and exclusive $\left(B \rightarrow D^{(\star)} l v\right)$ channels [3]. The latest world average values for $\left|\mathrm{V}_{\mathrm{cb}}\right|$ are listed in Table 1 . The analysis of kinematic spectra allows for comparisons of different new physics models. Especially the endpoints of these spectra are very sensitive to the couplings of new physics [4].

Measurement strategy The strategy for this kind of measurement for example for $B \rightarrow D l v$ would be to tag hadronically, such that one can calibrate the tag using semileptonic modes, for example $B \rightarrow X l v$. To extract the signal a binned maximum likelihood fit to the missing mass squared, $M_{\mathrm{miss}}^{2}$, can be performed. The yields then can be extracted in bins of kinematic variables, such as $w=\left(m_{B}^{2}+m_{D}^{2}-q^{2}\right) /\left(2 m_{B} m_{D}\right)$ [5]. Note that in the case of $B \rightarrow D^{\star} l v$ there are 4 kinematic variables to fit, preferably in 4-dimensional bins.

Table 1: Results of recent $\left|V_{c b}\right|$ measurements [6]. The discrepancy between inclusive and exclusive measurements is of the order of $2.5 \sigma$.

\begin{tabular}{rccl} 
Mode & $\times 10^{-3}$ & experimental & theory \\
\hline$D l v$ & 39.18 & \pm 0.94 & \pm 0.36 \\
$D^{\star} l v$ & 39.05 & \pm 0.47 & \pm 0.58 \\
$X_{c} l v$ & 42.11 & \pm 0.74 & (combined) \\
\hline
\end{tabular}

Projections for Belle II The expected experimental errors for Belle II are depicted in Table 2. These extractions depend on form factors obtained from lattice QCD. The experimental improvements are made in low momentum pion tracking, lepton identification and an increased hadronic tag efficiency.

Table 2: Projected experimental errors for Belle II [6]. For all of the observables we expect the uncertainties to reduce to the percent level.

\begin{tabular}{rcc} 
Mode & $\left|V_{c b}\right|$ & $R_{e / \mu}$ \\
\hline$B \rightarrow D^{\star} l v$ & $3 \% \rightarrow 1 \%$ & $5 \% \rightarrow 1 \%$ \\
$B \rightarrow D l v$ & $3 \% \rightarrow 1 \%$ & $\approx 6 \% \rightarrow 1 \%$ \\
\hline
\end{tabular}

\section{Measurement and projections for $B \rightarrow D^{(\star)} \tau \nu$ and $R_{\tau}$}

The semileptonic modes containing a $\tau$ lepton are of special interest as one can perform similar measurements of lepton flavour violation of the $\tau$ which might, due to its high mass, be a better candidate to search for new physics couplings. However, since the decay modes with a $\tau$ involve at least two neutrinos in the final state, the measurement becomes more challenging, as fake $D^{\star}, D^{\star \star}$ and escaping $K_{\mathrm{L}}^{0}$ are very challenging to veto. The quantity of interest in this channel is the ratio $R_{\tau}:=\mathscr{B}\left(B \rightarrow D^{(\star)} \tau \nu\right) / \mathscr{B}\left(B \rightarrow D^{(\star)} l v\right)$, where $l=e, \mu$. Only some previous measurements have shown agreement with the standard model [7]. However, the kinematic spectra should be measured with high precision such that new physics models including modified $\tau$ couplings can be ruled out. 
Measurement strategy The measurement strategy in this case would be to use for example the residual energy in the calorimeter, $E_{\mathrm{ECL}}$, or the missing mass squared, $m_{\mathrm{miss}}^{2}$, to find the signal and a machine learning technique, for example a Boosted Decision Tree to separate decays containing $\tau$ leptons from decays with $l=e$ or $l=\mu$. A simultaneous fit of the classifier output and the signal variable then can be used to extract the yields [8].

Projections for Belle II The Belle II projections for the ratio $R_{\tau}$ are depicted in Table 3. With the full dataset the limiting factor of the systematic uncertainties will be the determination of $D^{\star \star}$ backgrounds and fake $D^{\star}$ reconstructions.

Table 3: Expected Belle II projections for the measurement uncertainties of $R_{\tau}$ [6].

\begin{tabular}{ccccccc} 
& \multicolumn{3}{c}{$R_{\tau}(D)$} & \multicolumn{3}{c}{$R_{\tau}\left(D^{\star}\right)$} \\
$\int \mathscr{L} d t$ & stat & sys & total & stat & sys & total \\
\hline $0.7 \mathrm{ab}^{-1}$ & $14 \%$ & $6 \%$ & $16 \%$ & $6 \%$ & $3 \%$ & $7 \%$ \\
$5 \mathrm{ab}^{-1}$ & $5 \%$ & $3 \%$ & $6 \%$ & $2 \%$ & $2 \%$ & $3 \%$ \\
$50 \mathrm{ab}^{-1}$ & $2 \%$ & $3 \%$ & $3 \%$ & $1 \%$ & $2 \%$ & $2 \%$ \\
\hline
\end{tabular}

\section{Measurement of $B \rightarrow \pi l v, B \rightarrow \pi \tau v$ and projections for $\left|\mathrm{V}_{\mathrm{ub}}\right|$ and $R_{\pi}$}

The exclusive (for example $B \rightarrow \pi l v)$ and inclusive $\left(B \rightarrow X_{u} l v\right.$, where $X_{u}$ is any light meson containing a u-quark) determination of $\left|\mathrm{V}_{\mathrm{ub}}\right|$ show a similar discrepancy to that of $\left|\mathrm{V}_{\mathrm{cb}}\right|$. The extractions of $\left|V_{u b}\right|$ depend on form factors obtained from lattice QCD and will be limited by the uncertainties on the calculation of these parameters once the full Belle II dataset is available. Belle II will also be able to perform measurements of $B \rightarrow \pi \tau v$ for which so far only an upper limit of $\mathscr{B}\left(B^{0} \rightarrow \pi^{-} \tau^{+} v_{\tau}\right)<2.5 \times 10^{-4}$ could be set [9]. Once this measurement can be performed the ratio $R_{\pi}:=\mathscr{B}(B \rightarrow \pi \tau v) / \mathscr{B}(B \rightarrow \pi l v)$, where $l=e, \mu$ can be measured with high precision.

Measurement strategy The measurement strategy for $B \rightarrow \pi \tau \nu$ is similar to the channels containing charm mesons using a hadronic or semileptonic tagging technique, see for example [10]. For the $\tau$ channel the $\tau$ is reconstructed in $\tau \rightarrow l v \nu$ and $\tau \rightarrow \pi \nu$, the signal is measured in either $E_{\mathrm{ECL}}$ or $m_{\text {miss }}^{2}$ and a BDT can be used to separate off the decays of $B \rightarrow \pi l v$. The main challenge will lie in controlling the backgrounds from $B \rightarrow D^{(\star)} l \nu$ and $B \rightarrow D^{(\star)} \tau \nu$.

Projections for Belle II The expected projections of the total expected uncertainties of the $\left|V_{\mathrm{ub}}\right|$ determination are depicted in figure 1 . The projected uncertainties for $R_{\pi}$ are tabled in Table 4.

Table 4: Belle II projections for the measurement of $R_{\pi}$ for different dataset sizes [6].

\begin{tabular}{cc}
$\int \mathscr{L} d t$ & $R_{\pi}$ \\
\hline $5 \mathrm{ab}^{-1}$ & $0.64 \pm 0.23$ \\
$50 \mathrm{ab}^{-1}$ & $0.64 \pm 0.09$ \\
\hline
\end{tabular}




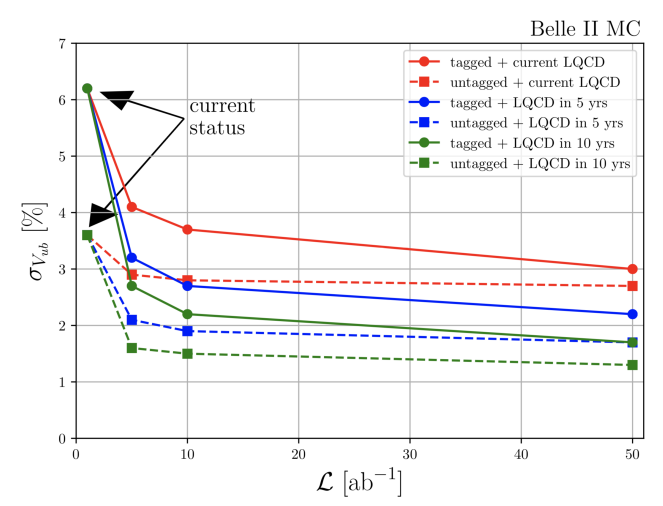

Figure 1: Evolution of the total uncertainty on $\left|V_{\mathrm{ub}}\right|$ with the growing Belle II data set for various tag scenarios, based on a full Belle II Monte Carlo simulation and the expected lattice QCD improvements [6].

\section{Conclusion}

The Belle II experiment has started collecting data, by 2025 we expect to have the full dataset of $50 \mathrm{ab}^{-1}$ available. The first new results for semileptonic B-decays can be be published using a sample size of $5 \mathrm{ab}^{-1}$ which will be available by the end of the year 2020. The expected experimental uncertainties for the measurement of $\left|V_{c b}\right|$ will decrease to a level of $1 \%$ using the full dataset. This should suffice to resolve the tension of inclusive and exclusive measurements of this quantity. The ratio $R_{e / \mu}$ can be measured to a precision of $1 \% . R_{\tau}$ can be measured to a level of $3 \%$ in $B \rightarrow D \tau \nu$ and $2 \%$ in $B \rightarrow D^{\star} \tau \nu$. The measurement of $\left|V_{u b}\right|$ will be possible with an uncertainty of less than $3 \%$, which should allow to understand the current tensions between inclusive and exclusive measurements. The determination of $R_{\pi}$ will be possible to the sub percent level.

\section{References}

[1] T. Abe et al. "Belle II Technical Design Report". In: (2010). arXiv: 1011.0352.

[2] Thomas Keck et al. "The Full Event Interpretation - An exclusive tagging algorithm for the Belle II experiment”. In: (2018). arXiv: 1807.08680.

[3] Pietro Colangelo and Fulvia De Fazio. "Tension in the inclusive versus exclusive determinations of $\left|V_{c b}\right|$ : a possible role of new physics". In: Phys. Rev. D95.1 (2017), p. 011701. DOI: 10.1103/PhysRevD.95.011701. arXiv: 1611.07387.

[4] Martin Jung and David M. Straub. "Constraining new physics in $b \rightarrow c \ell v$ transitions". In: (2018). arXiv: 1801.01112.

[5] Y. Sato et al. "Measurement of the branching ratio of $\bar{B}^{0} \rightarrow D^{*+} \tau^{-} \bar{v}_{\tau}$ relative to $\bar{B}^{0} \rightarrow$ $D^{*+} \ell^{-} \bar{v}_{\ell}$ decays with a semileptonic tagging method". In: Phys. Rev. D94.7 (2016), p. 072007. DOI: $10.1103 /$ PhysRevD.94.072007. arXiv: 1607.07923.

[6] E. Kou et al. “The Belle II Physics Book”. In: (2018). arXiv: 1808.10567. 
[7] R. Aaij et al. "Test of Lepton Flavor Universality by the measurement of the $B^{0} \rightarrow D^{*-} \tau^{+} v_{\tau}$ branching fraction using three-prong $\tau$ decays". In: Phys. Rev. D97.7 (2018), p. 072013. DOI: 10.1103/PhysRevD.97.072013. arXiv: 1711.02505.

[8] Y. Sato et al. "Measurement of the branching ratio of $\bar{B}^{0} \rightarrow D^{*+} \tau^{-} \bar{v}_{\tau}$ relative to $\bar{B}^{0} \rightarrow$ $D^{*+} \ell^{-} \bar{v}_{\ell}$ decays with a semileptonic tagging method". In: Phys. Rev. D94.7 (2016), p. 072007. DOI: 10.1103 /PhysRevD. 94.072007 . arXiv: 1607.07923.

[9] P. Hamer et al. "Search for $B^{0} \rightarrow \pi^{-} \tau^{+} v_{\tau}$ with hadronic tagging at Belle". In: Phys. Rev. D93.3 (2016), p. 032007. DOI: 10.1103 / PhysRevD . 93.032007 . arXiv: 1509 . 06521.

[10] A. Sibidanov et al. "Study of Exclusive $B \rightarrow X_{u} \ell v$ Decays and Extraction of $\left\|V_{u b}\right\|$ using Full Reconstruction Tagging at the Belle Experiment”. In: Phys. Rev. D88.3 (2013), p. 032005. DOI: $10.1103 /$ PhysRevD .88 .032005 . arXiv: 1306.2781. 